\title{
The initial experience of dual port laparoscopy-assisted total gastrectomy as a reduced port surgery for total gastrectomy
}

\author{
Hideki Kawamura $\cdot$ Toshiro Tanioka $\cdot$ Mariko Kuji • \\ Munenori Tahara $\cdot$ Masahiro Takahashi
}

Received: 17 August 2012/ Accepted: 2 November 2012/Published online: 21 November 2012

(c) The International Gastric Cancer Association and The Japanese Gastric Cancer Association 2012

\begin{abstract}
Reduced port surgery (RPS), in which fewer ports are used than that in a conventional laparoscopic procedure, is becoming increasingly popular for various surgeries. However, the application of RPS to the field of gastrectomy is still underdeveloped. We started laparoscopy-assisted total gastrectomy through an umbilical port plus another $5 \mathrm{~mm}$ port (dual port laparoscopy-assisted total gastrectomy: DP-LATG) as an RPS for laparoscopyassisted total gastrectomy (LATG). A SILS ${ }^{\mathrm{TM}}$ port was inserted into an umbilical incision, while another $5 \mathrm{~mm}$ port was inserted at the right flank region. We performed DP-LATG on ten early gastric cancer cases consecutively from May 2011 onwards, with the surgeries all performed by a single surgeon. The results of DP-LATG were compared with the resuls of ten conventional LATGs (C-LATGs) that were performed between March 2010 and April 2011. There were no significant differences in the mean operation time (DP-LATG, $253.0 \pm 26.8 \mathrm{~min}$; C-LATG, $235.5 \pm 20.6 \mathrm{~min} ; p=0.119)$, mean blood loss $(33.4 \pm 23.7,39.8 \pm 60.4 \mathrm{~mL}, p=0.759)$, and number of lymph nodes dissected $(31.6 \pm 12.3, \quad 40.9 \pm 18.7$, $p=0.205)$. There were no intraoperative complications, there was no need for additional ports, and there were no conversions to open surgery nor postoperative complications in the DP-LATG cases. We successfully and safely performed DP-LATG without incurring any notable
\end{abstract}

H. Kawamura $(\bowtie) \cdot$ T. Tanioka · M. Kuji · M. Tahara ·

M. Takahashi

Department of Surgery, JA Sapporo Kosei Hospital,

N3E8, Chuo-ku, Sapporo 060-0033, Japan

e-mail: h.kawamura@ja-hokkaidoukouseiren.or.jp differences from C-LATG in terms of operation time, blood loss, and number of lymph nodes dissected.

Keywords Laparoscopy - Total gastrectomy ·

Single port · Reduced port · Gastric cancer

\section{Introduction}

In recent years, reduced port surgery (RPS), including single port surgery (SPS), in which fewer ports are used than in conventional procedures, has become increasingly popular in various surgical fields [1-14]. There have been reports describing the application of RPS to laparoscopic sleeve gastrectomy for morbid obesity or laparoscopic partial gastrectomy for gastrointestinal stromal tumors, and the use of RPS has recently also been reported in laparoscopy-assisted distal gastrectomy for gastric cancer [1, 7, 15-17]. However, to date, the use of RPS in laparoscopyassisted total gastrectomy (LATG), a procedure that requires high levels of technical expertise, has not been reported.

We started reduced port laparoscopy-assisted distal gastrectomy through an umbilical port and an additional port (DP-LADG: dual port laparoscopy-assisted distal gastrectomy) for early gastric cancer located in the lower or middle part of the stomach from December 2009, and reported the acceptability of DP-LADG [17]. As the next step, we performed laparoscopy-assisted total gastrectomy through an umbilical port plus another $5 \mathrm{~mm}$ port (dual port laparoscopy-assisted total gastrectomy: DP-LATG) as an RPS for LATG in ten consecutive early gastric cancer patients from May 2011 onwards. Here, we report the procedure and the operative outcomes of these cases in comparison with the outcomes for patients receiving 
conventional laparoscopy-assisted total gastrectomy (C-LATG).

\section{Materials and methods}

All DP-LATGs were performed by a single surgeon who had experienced more than 400 cases of laparoscopic gastrectomy (including 110 cases of C-LATG) and 30 cases of DP-LADG as the operator. The indication for DPLATG was preoperative clinical stage IA gastric cancer located in the upper part of the stomach without previous upper abdominal surgery. All patients underwent D1 (+nos. 8a, 9, 11p, 11d) lymph node dissection. Informed consent for the surgery was obtained from all patients. The patients were told that the surgeon had adequate experience of laparoscopic gastrectomy and reduced port surgery, but that DP-LATG is not an established procedure, and that it was possible to convert to C-LATG.

Gastric cancer is described in accordance with the Classification of Gastric Carcinoma (third edition) and the Japanese
Gastric Cancer Treatment Guidelines 2010, as drawn up by the Japanese Gastric Cancer Association [18, 19].

\section{Surgical procedure for DP-LATG}

\section{Port setting and devices used in DP-LATG}

The patient was placed in Fowler's position with the legs abducted. A SILS ${ }^{\mathrm{TM}}$ port (Covidien Ltd., Hamilton, Bermuda) with three in-built trocars was inserted into an umbilical incision, while another $5 \mathrm{~mm}$ port was inserted in the right flank region (Fig. 1a). Figure $1 \mathrm{~b}$ shows the wound of the Fig. 1a patient one month after surgery. A $5 \mathrm{~mm}$ flexible scope was inserted through the $5 \mathrm{~mm}$ trocar at the extreme caudal position of the SILS ${ }^{\mathrm{TM}}$ port for the duration of the procedure. The surgeon used either two trocars in the SILS ${ }^{\mathrm{TM}}$ port or one trocar in the SILS ${ }^{\mathrm{TM}}$ port and one in the right flank port. The surgeon stood between the patient's legs in the former situation and on the right side of the patient in the latter (Fig. 1c, d). The assistant used the remaining trocar to provide support. Activating laparoscopic coagulating shears
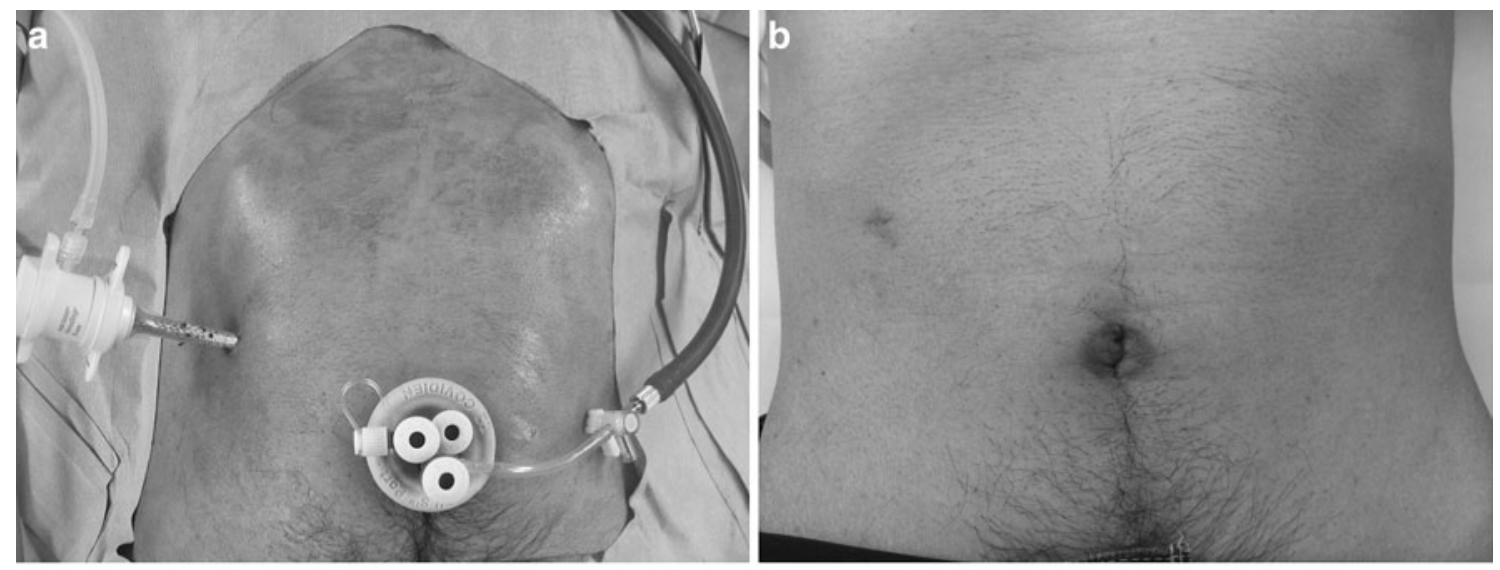

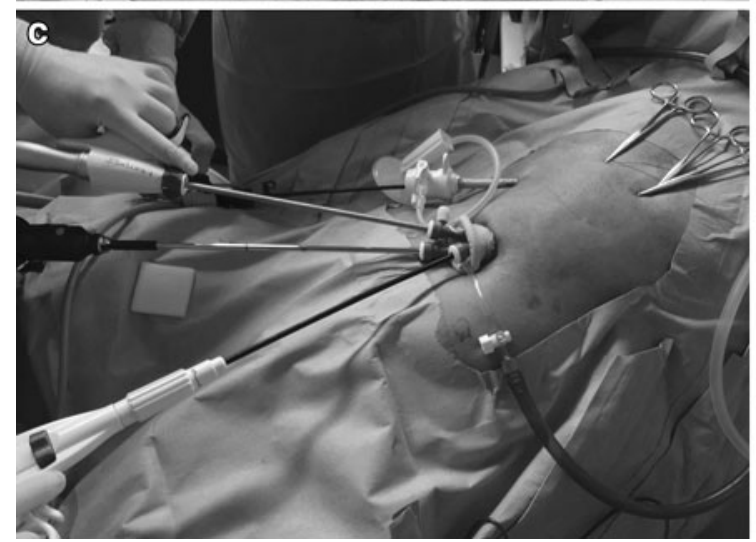

Fig. 1 Port setting of and wound from dual-port laparoscopy-assisted total gastrectomy. a A SILS ${ }^{\mathrm{TM}}$ port was inserted into an umbilical incision, while another $5 \mathrm{~mm}$ port was inserted in the right flank region. b Wound after one month. c The surgeon stood between the

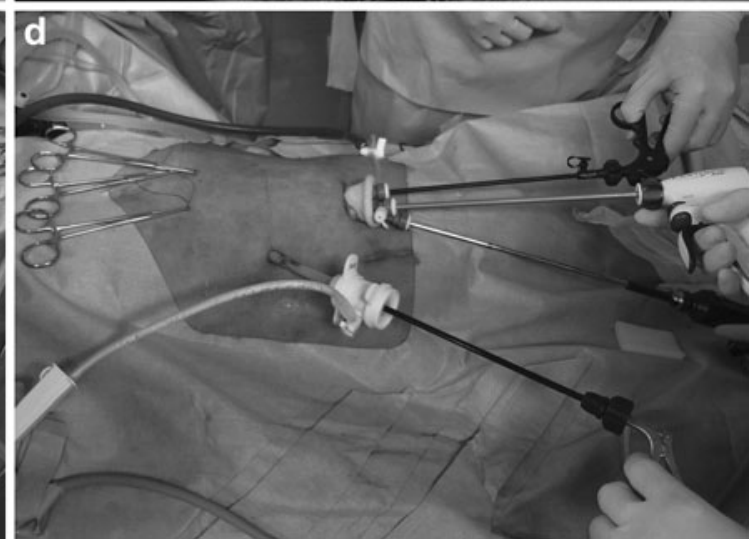

patient's legs and used two trocars in the SILS ${ }^{\mathrm{TM}}$ port. $\mathbf{d}$ The surgeon stood on the right side of the patient and used one trocar in the SILS $^{\mathrm{TM}}$ port and one in the right flank port 
(LCS) were used for dissection. A straight and a curved type grasper (Roticulator ${ }^{\mathrm{TM}}$ Endo Grasp ${ }^{\mathrm{TM}}$; Covidien Ltd.) were used to grasp the tissue.

The steps involved in the DP-LATG procedure were as follows:

\section{Surgery of the greater curvature side}

The surgeon stood between the patient's legs and used the upper two $5 \mathrm{~mm}$ trocars in the SILS ${ }^{\mathrm{TM}}$ port. The assistant grasped and raised the stomach wall using the grasper inserted through the right flank port. The operator subsequently dissected the omentum as well as the left and right gastroepiploic vessels (Fig. 2a, b).

\section{Transection of the duodenum}

The surgeon moved to the right side of the patient after manipulating the greater curvature side. The left upper $5 \mathrm{~mm}$ trocar of the SILS ${ }^{\mathrm{TM}}$ port was replaced with the $12 \mathrm{~mm}$ trocar to insert the endolinear stapler used for transection of the duodenum. After performing transection of the duodenum, the $12 \mathrm{~mm}$ port was replaced with the $5 \mathrm{~mm}$ trocar.

3. Surgery of the lesser curvature side and lymph node dissection of the superior side of the pancreas
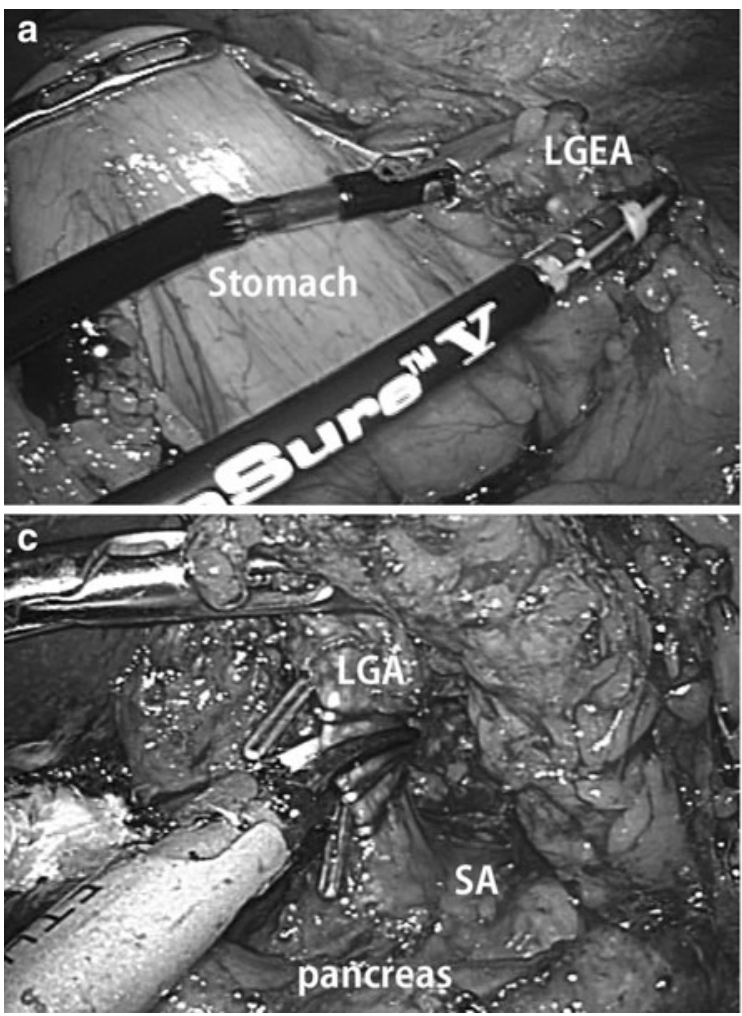

Fig. 2 Steps involved in dual-port laparoscopy-assisted total gastrectomy. a Lymph node dissection around the left gastroepiploic artery. b Lymph node dissection around the right gastroepiploic vein. c Lymph node dissection of the superior side of the pancreas (nos. 8a,

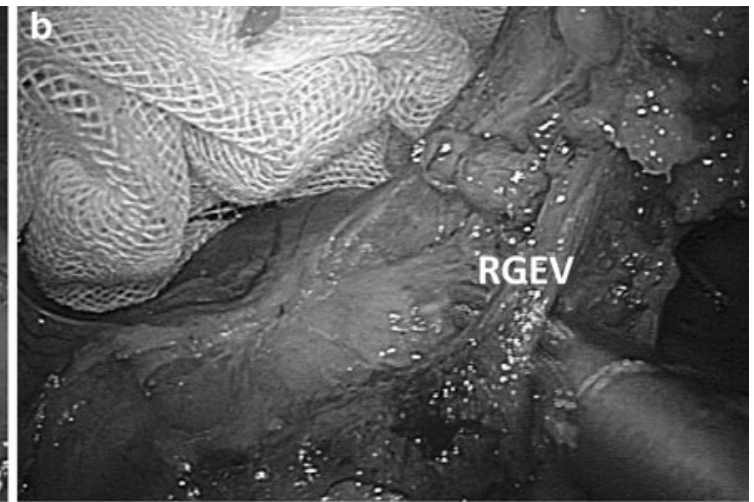

The surgeon used the right upper or the left upper trocar of the SILS ${ }^{\mathrm{TM}}$ port for the LCS and the right flank port for the grasper. The assistant used the remaining trocar of the SILS $^{\mathrm{TM}}$ port for retraction. The right gastric artery/vein, lesser omentum, lymph node of the superior side of the pancreas (nos. 8a, 11p), and left gastric vein/artery were dissected sequentially (Fig. 2c).

4. Dissection of the esophagus and lymph node dissection along the distal splenic artery

The left upper $5 \mathrm{~mm}$ trocar of the SILS ${ }^{\mathrm{TM}}$ port was replaced with the $12 \mathrm{~mm}$ trocar to insert the endolinear stapler, and the esophagus was dissected. To ease lymph node dissection along the distal splenic artery (no. 11d), this dissection was performed after amputating the esophagus, half-mobilizing the cranial side of the distal pancreas and spleen (Fig. 2d).

5. Extraction of the stomach and Y-limb anastomosis

The SILS ${ }^{\mathrm{TM}}$ port was removed, and a wound protector was attached to the umbilical incision. The stomach was extracted from the umbilical incision, and a Y-limb anastomosis was created directly.

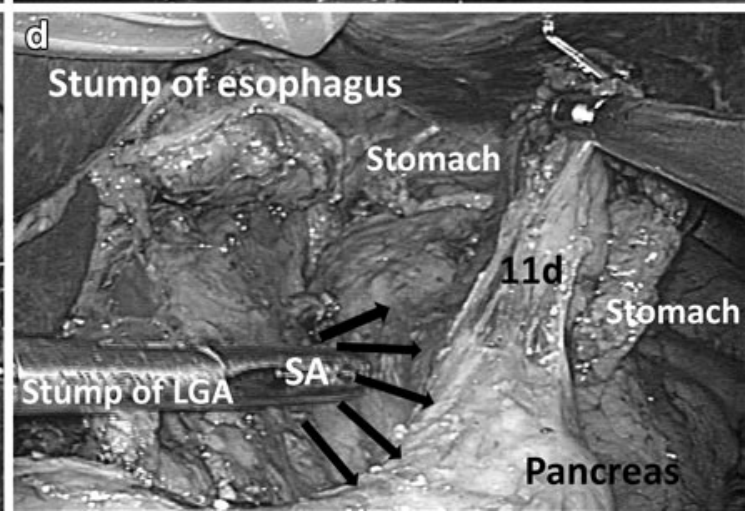

$11 \mathrm{p})$ and around the left gastric vein/artery. $\mathbf{d}$ Lymph node dissection along the distal splenic artery. $L G E A$ left gastroepiploic artery, $R G E V$ right gastroepiploic vein, $L G A$ left gastric artery, $S A$ splenic artery 


\section{Esophagojejunostomy}

The SILS ${ }^{\mathrm{TM}}$ port was reattached, and esophagojejunostomy was performed laparoscopically by side-to side anastomosis using an endolinear stapler (Fig. 3a). Esophagojejunostomy using an endolinear stapler as well as a circular stapler has been applied in C-LATG, but an endolinear stapler rather than a circular stapler would be suitable for insertion through an umbilical multiport. The incision used to insert the endolinear stapler was closed using interrupted sutures. Figure $3 b$ shows the final state after lymph node dissection and anastomosis. A drain was inserted via the right flank port incision.

\section{A knack for handling instruments thorough an umbilical multiport}

There is a corresponding disadvantage to SPS because it lacks free movement, as there is conflict among forceps, a cutting device, and a scope. Therefore, the key to smoothly performing SPS lies in understanding the formation in which the shaft conflict is minimized (i.e., the formation in which there is the highest degree of freedom of movement). A mechanical analysis demonstrated that a formation in which the three instruments rotate clockwise or counterclockwise, called the "rotation formation," is the ideal one for SPS, with its excellent instrument operability (Fig. 3c) [20]. Utilizing the rotation formation, we were able to perform DP-LATG using almost the same steps as C-LATG under dual port conditions.

\section{Comparison of the outcomes of the DP-LATG group} with the outcomes of an independent C-LATG group

To compare the results obtained with DP-LATG to those provided by C-LATG, we prepared a group of ten consecutive early gastric cancer cases who underwent C-LATG before starting DP-LATG (between March 2010 and April 2011), where the surgeries were all performed by the same surgeon. The steps involved in the C-LATG procedure, the reconstruction, and the degree of lymph node dissection performed in this group were basically the same as those for DP-LATG, except that the dual port setting is used for DP-LATG.

\section{Statistical analysis}

The $t$ test and Pearson's chi-squared test were used for statistical investigations. SPSS 16.0 for Windows (SPSS
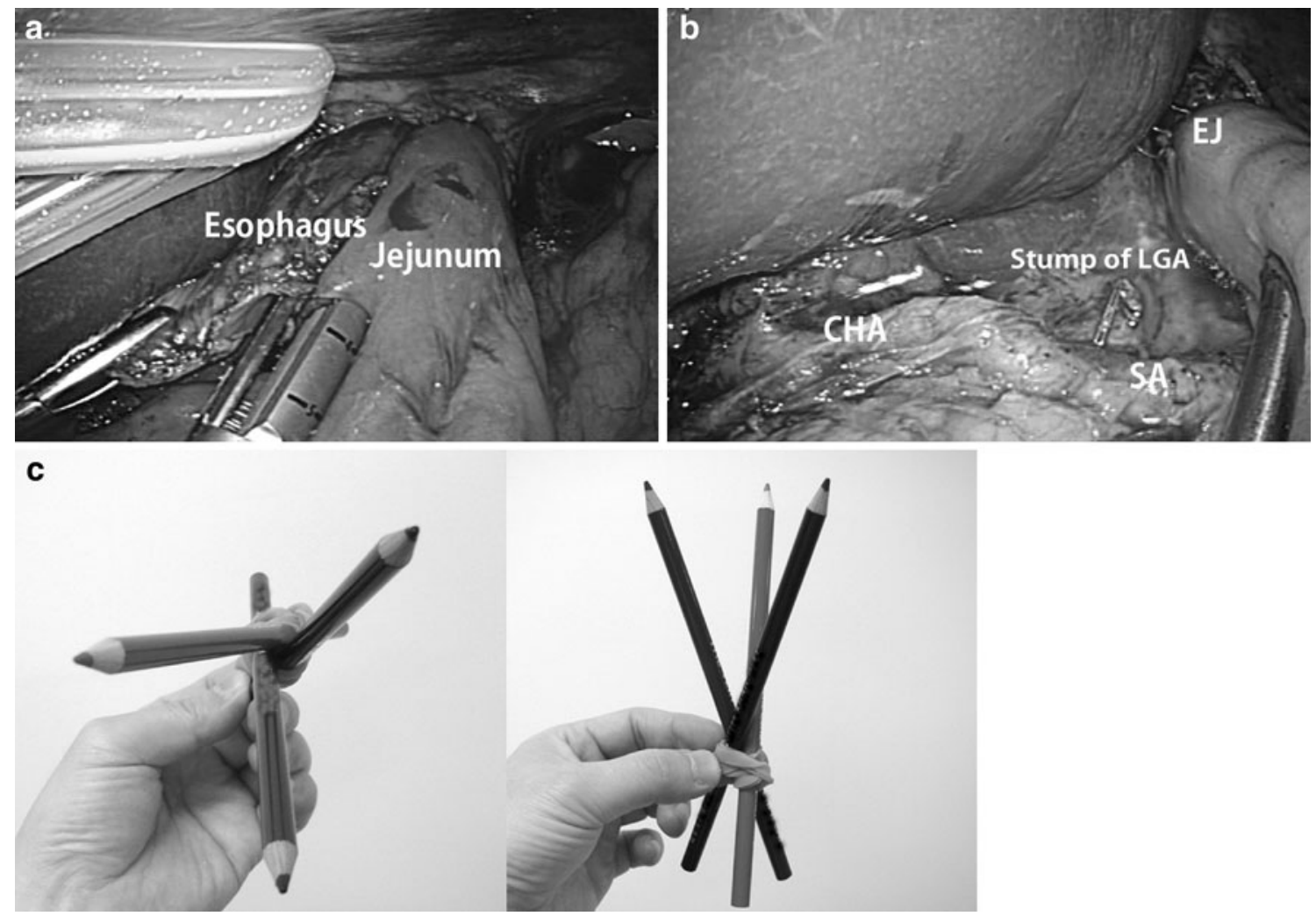

Fig. 3 a Esophagojejunostomy using an endolinear stapler. b Final state after lymph node dissection and anastomosis. c The rotation formation. Head-on view of the rotation position (left) and side view of the rotation formation (right). CHA common hepatic artery, EJ esophagojejununostomy, $S A$ splenic artery 
Inc., Chicago, IL, USA) statistical software was used for the statistical analyses. Differences were considered significant at a $P$ value of $<0.05$.

\section{Results}

Patient details are listed in Table 1. All patients successfully underwent DP-LATG. The mean age was $65.7 \pm 6.0$ years. Seven patients were male and three were female. Three were classified as American Society of Anesthesiologists (ASA) class 1 and seven patients were ASA class 2. The patients' mean body mass index was $23.8 \pm 1.8$. There was no significant difference in patient background between the DP-LATG group and the C-LATG group (Table 2). The mean operation time for the DP-LATG group $(253.0 \pm 26.8 \mathrm{~min})$ was $17.5 \mathrm{~min}$ longer than that for the C-LATG group $(235.5 \pm 20.6 \mathrm{~min})$, although this was not a significant difference. There was no significant difference in mean blood loss $(33.4 \pm 23.7$, $39.8 \pm 60.4 \mathrm{~mL}$ ) or number of dissected lymph nodes $(31.6 \pm 12.3,40.9 \pm 18.7)$ between two groups. There was also no significant difference in pStage between two groups. There were no intraoperative complications, there was no need for additional ports, and there were no conversions to open surgery nor postoperative complications in the DP-LATG group (Table 3).

\section{Discussion}

Performing gastrectomy by RPS requires familiarity with not only gastrectomy and laparoscopic techniques but also single port surgery. Furthermore, laparoscopic total gastrectomy is one of the most difficult of the laparoscopic surgical procedures. As a result, RPS for laparoscopic total gastrectomy has not been previously reported. In this report, all ten cases successfully underwent DP-LATG without any trouble, due to our adequate experience of C-LATG and reduced port distal gastrectomy. In our subjective opinion, before beginning reduced port LATG, the surgeon must not only have enough skill to perform C-LATG but must have experienced at least 20 cases of reduced port LADG.

The most important merit of RPS is cosmesis [3, 4, 6, 9, 11, 13]. Therefore, this cosmetic merit must be retained as long as possible when performing RPS for total gastrectomy. Single port surgery is performed through an umbilical port that usually includes three trocars. The laparoscope is passed through one trocar, and devices manipulated by the surgeon's hands are inserted into the other two trocars. In laparoscopic gastrectomy, unfolding of the omentum and tissue grasping or retraction by the

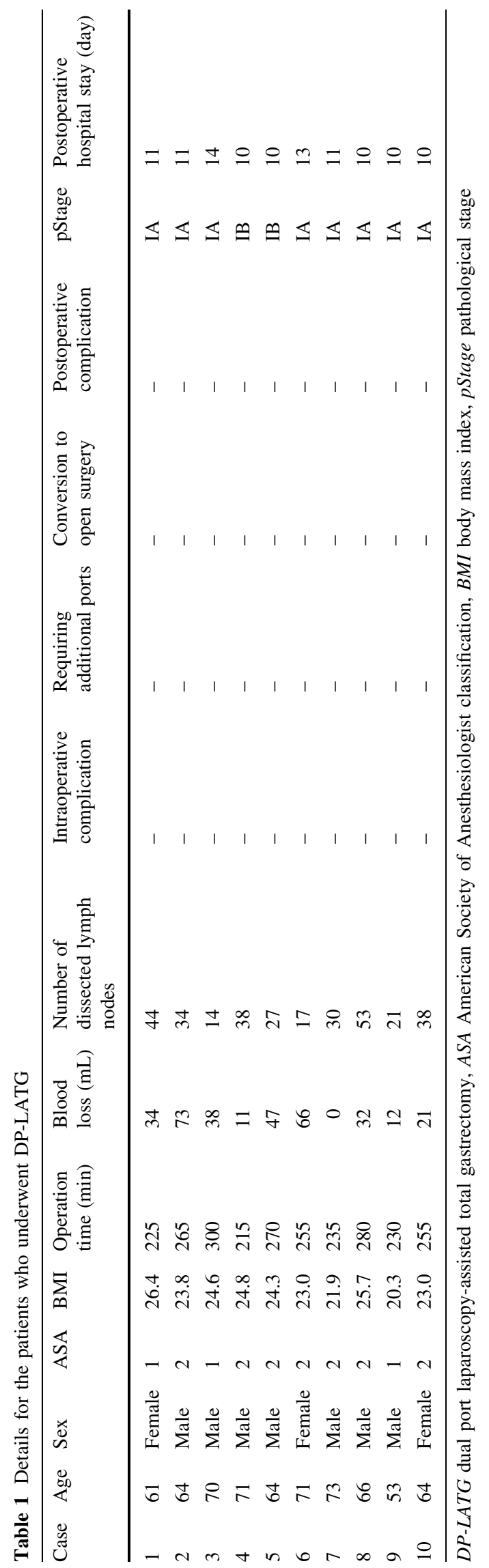


Table 2 Comparison of the patient background for the DP-LATG group with that for the C-LATG group

\begin{tabular}{llll}
\hline Authors & $\begin{array}{l}\text { DP-LATG } \\
(n=10)\end{array}$ & $\begin{array}{l}\text { C-LATG } \\
(n=10)\end{array}$ & $P$ \\
\hline Age & $65.7 \pm 6.0$ & $61.7 \pm 16.5$ & 0.481 \\
$\begin{array}{l}\text { Sex (male/ } \\
\text { female) }\end{array}$ & $7 / 3$ & $5 / 5$ & 0.650 \\
BMI & $23.8 \pm 1.8$ & $23.6 \pm 3.5$ & 0.862 \\
ASA $(1 / 2 / 3)$ & $3 / 7 / 0$ & $3 / 6 / 1$ & 0.584 \\
\hline
\end{tabular}

$D P$ - $L A T G$ dual port laparoscopy-assisted total gastrectomy, $C$ - $L A T G$ conventional laparoscopy-assisted total gastrectomy, $B M I$ body mass index, ASA American Society of Anesthesiologist classification

Table 3 Comparison of the results obtained using DP-LATG and C-LATG

\begin{tabular}{|c|c|c|c|}
\hline Authors & $\begin{array}{l}\text { DP-LATG } \\
(n=10)\end{array}$ & $\begin{array}{l}\text { C-LATG } \\
(n=10)\end{array}$ & $P$ \\
\hline Operation time (min) & $253.0 \pm 26.8$ & $235.5 \pm 20.6$ & 0.119 \\
\hline Blood loss (mL) & $33.4 \pm 23.7$ & $39.8 \pm 60.4$ & 0.759 \\
\hline $\begin{array}{l}\text { Number of dissected } \\
\text { lymph nodes }\end{array}$ & $31.6 \pm 12.3$ & $40.9 \pm 18.7$ & 0.205 \\
\hline pStage (IA/IB/II) & $8 / 2 / 0$ & $6 / 3 / 1$ & 0.476 \\
\hline \multicolumn{4}{|c|}{ Intraoperative complications } \\
\hline+ & 0 & 0 & \\
\hline- & 10 & 10 & \\
\hline \multicolumn{4}{|c|}{ Postoperative complications } \\
\hline+ & 0 & $\begin{array}{l}1 \text { (abdominal } \\
\text { abscess) }\end{array}$ & 1.000 \\
\hline- & 10 & 9 & \\
\hline
\end{tabular}

$D P-L A T G$ dual port laparoscopy-assisted total gastrectomy, $C$-LATG conventional laparoscopy-assisted total gastrectomy, pStage pathological stage

assistant are always required in order to perform most parts of the procedure, so the use of an umbilical port alone is not feasible. We added one supportive port in the right flank region to solve this problem, and no patient required the addition of other ports in this study. This result suggests that one additional port is technically sufficient to perform RPS for LATG in many cases. However, because we had experienced a DP-LADG case with upper abdominal adhesion in which additional ports were required, we excluded cases with previous upper abdominal surgery from the indication for DP-LATG. In this series, we did not consider BMI for the indication, and the most obese (BMI 29.9) case successfully underwent DP-LATG without an additional port, however, DP-LATG should also be avoided in morbidly obese cases. In any case, since reduced port surgery has not demonstrated any advantages beyond cosmesis so far, the surgeon should not hesitate to add a port if necessary in difficult cases.
In this study, all patients underwent DP-LATG without the need for any additional ports or to convert to open surgery. However, this would not be advantageous if the operation time was much longer or the amount of blood loss was much greater than those associated with C-LATG. The reported operation time, blood loss, and number of dissected lymph nodes in C-LATG were 187-304 min, 10-334 mL, and 26-48.5, respectively [21-25]. Our results for the operation time $(253.0 \pm 26.8 \mathrm{~min})$, blood loss $(33.4 \pm 23.7 \mathrm{~mL})$, and the number of dissected lymph nodes $(31.6 \pm 12.3)$ were within the ranges reported for C-LATG $[21,22,25]$. In the comparison between DP-LATG and C-LATG performed at our institute, there were no significant differences in the results. Furthermore, there were no intra- or postoperative complications. We consider that DP-LATG is an acceptable procedure with regard to operation time, safety, and accuracy. However, we must again mention that these results were premised on our adequate experience of C-LATG and reduced port distal gastrectomy, our understanding of the rotation formation, and one effective additional port.

Patients who underwent DP-LATG had an almost invisible umbilical scar and one $5 \mathrm{~mm}$ long wound, and this may have resulted in a better mental status for them than that for patients who underwent C-LATG. To make a definitive conclusion about this suggestion, an assessment of quality of life (including mental status) would be required. Furthermore, laparoscopic total gastrectomy is widely recognized as a less invasive surgery, but it is unknown whether RPS for total gastrectomy is less invasive than conventional laparoscopic gastrectomy [21-25]. Also, lately, procedures using 2 or $3 \mathrm{~mm}$ diameter forceps are gradually increasing in popularity for various surgeries. Would the RPS for LATG be superior to LATG performed by 2 or $3 \mathrm{~mm}$ ports? As the next step, we plan to examine these subjects. This small study represents a feasibility study for the design of a prospective study.

In conclusion, we successfully and safely performed DP-LATG as a RPS for LATG, and found that there were no large differences between DP-LATG and C-LATG in operation time, blood loss, or lymph node dissection.

\section{References}

1. Reavis KM, Hinojosa MW, Smith BR, Nguyen NT. Single-laparoscopic incision transabdominal surgery sleeve gastrectomy. Obes Surg. 2008;18:1492-4.

2. Nguyen NT, Hinojosa MW, Smith BR, Reavis KM. Single laparoscopic incision transabdominal (SLIT) surgery-adjustable gastric banding: a novel minimally invasive surgical approach. Obes Surg. 2008;18:1628-31.

3. Gill IS, Canes D, Aron M, Haber GP, Goldfarb DA, Flechner S, et al. Single port transumbilical (E-NOTES) donor nephrectomy. J Urol. 2008;180:637-41. 
4. Teixeira J, McGill K, Binenbaum S, Forrester G. Laparoscopic single-site surgery for placement of an adjustable gastric band: initial experience. Surg Endosc. 2009;23:1409-14.

5. Fader AN, Escobar PF. Laparoendoscopic single-site surgery (LESS) in gynecologic oncology: technique and initial report. Gynecol Oncol. 2009;114:157-61.

6. Hernandez JM, Morton CA, Ross S, Albrink M, Rosemurgy AS. Laparoendoscopic single site cholecystectomy: the first 100 patients. Am Surg. 2009;75:681-5 (discussion 685-686).

7. Saber AA, Elgamal MH, Itawi EA, Rao AJ. Single incision laparoscopic sleeve gastrectomy (SILS): a novel technique. Obes Surg. 2008;18:1338-42.

8. Goel RK, Kaouk JH. Single port access renal cryoablation (SPARC): a new approach. Eur Urol. 2008;53:1204-9.

9. Remzi FH, Kirat HT, Kaouk JH, Geisler DP. Single-port laparoscopy in colorectal surgery. Colorectal Dis. 2008;10:823-6.

10. Desai MM, Rao PP, Aron M, Pascal-Haber G, Desai MR, Mishra $\mathrm{S}$, et al. Scarless single port transumbilical nephrectomy and pyeloplasty: first clinical report. BJU Int. 2008;101:83-8.

11. Hong TH, You YK, Lee KH. Transumbilical single-port laparoscopic cholecystectomy: scarless cholecystectomy. Surg Endosc. 2009;23:1393-7.

12. Langwieler TE, Nimmesgern T, Back M. Single-port access in laparoscopic cholecystectomy. Surg Endosc. 2009;23:1138-41.

13. Tacchino R, Greco F, Matera D. Single-incision laparoscopic cholecystectomy: surgery without a visible scar. Surg Endosc. 2009;23:896-9.

14. Barbaros U, Dinccag A. Single incision laparoscopic splenectomy: the first two cases. J Gastrointest Surg. 2009;13:1520-3.

15. Saber AA, El-Ghazaly TH. Early experience with SILS port laparoscopic sleeve gastrectomy. Surg Laparosc Endosc Percutan Tech. 2009;19:428-30.

16. Omori T, Oyama T, Akamatsu H, Tori M, Ueshima S, Nishida T. Transumbilical single-incision laparoscopic distal gastrectomy for early gastric cancer. Surg Endosc. 2011;25:2400-4.
17. Kawamura H, Tanioka T, Funakoshi T, Takahashi M. Dual-ports laparoscopy-assisted distal gastrectomy compared with conventional laparoscopy-assisted distal gastrectomy. Surg Laparosc Endosc Percutan Tech. 2011;21:429-33.

18. Japanese Gastric Cancer Association. Japanese classification of gastric carcinoma, 3rd English edn. Gastric Cancer. 2011;14: 101-12.

19. Japanese Gastric Cancer Association. Japanese gastric cancer treatment guidelines 2010. Gastric Cancer. 2011;14:113-23.

20. Kawamura H, Ishii C. Mechnical analysis of the formation of forceps and scope for single-port laparoscopic surgery. Surg Laparosc Endosc Percutan Tech. 2012;22:e168-74.

21. Usui S, Yoshida T, Ito K, Hiranuma S, Kudo S, Iwai T. Laparoscopy-assisted total gastrectomy for early gastric cancer: comparison with conventional open total gastrectomy. Surg Laparosc Endosc Percutan Tech. 2005;15:309-14.

22. Huscher CG, Mingoli A, Sgarzini G, Brachini G, Binda B, Paola $\mathrm{MD}$, et al. Totally laparoscopic total and subtotal gastrectomy with extended lymph node dissection for early and advanced gastric cancer: early and long-term results of a 100-patient series. Am J Surg. 2007;194:839-44.

23. Tanimura S, Higashino M, Fukunaga Y, Kishida S, Ogata A, Fujiwara Y, et al. Laparoscopic gastrectomy with regional lymph node dissection for upper gastric cancer. $\mathrm{Br} \mathrm{J}$ Surg. 2007;94:204-7.

24. Topal B, Leys E, Ectors N, Aerts R, Penninckx F. Determinants of complications and adequacy of resection in laparoscopic versus open total gastrectomy for adenocarcinoma. Surg Endosc. 2008;22:980-4.

25. Mochiki E, Toyomasu Y, Ogata K, Andoh H, Ohno T, Aihara R, et al. Laparoscopically assisted total gastrectomy with lymph node dissection for upper and middle gastric cancer. Surg Endosc. 2008;22:1997-2002. 